\title{
Case Study for Shuruchi Restaurant Queuing Model
}

\author{
Md. Al-Amin Molla \\ Md. Al-Amin Molla, Lecturer, Department of Natural Sciences, Daffodil International University, \\ Dhaka, Bangladesh
}

\begin{abstract}
Waiting lines and service systems are indispensable parts of our daily life. Every restaurant would like to avoid losing their desired customers due to a long wait in the queue. Providing more waiting chairs only, would not solve a problem when the customer withdraw this restaurant and go to another. We think service time need to be improved, which shows to manage the system and improve the queuing situation, we can apply queuing model. This paper aims to show the single channel queuing model M/M/l. We have obtained the one month daily customers data from a restaurant, named Shuruchi at Savar in Dhaka city. Using Little's theorem and the single channel waiting line model $M / M / 1$. We have determined the arrival rate $\lambda$, service rate $\mu$, the utilization rate $\rho$ and the average waiting time in the queue before getting service. We have also determined the probability of impatient customers to ignore the system. At Shuruchi restaurant, the arrival rate is 1.43 customers per minutes (cpm) and the service rate is 1.45 customers per minutes (cpm), the average number of customers is 64, customers waiting time is 14 minutes and the utilization rate is 0.98 . We have discussed about the benefits of applying queuing model to a busy restaurant in our conclusion part.
\end{abstract}

Keywords: Queue, Little's theorem, restaurant, M/M/1 queuing model, waiting times.

\section{Introduction}

Queuing theory is the mathematical study of queues or waiting lines. Queues or waiting lines are familiar phenomena to all. This theory is used to model and predict waiting times and the number of customers arrival. This paper uses queuing theory to study the waiting lines in Shuruchi restaurant at Savar in Dhaka. It is the busiest restaurant in Savar. It provides 10 tables of 6 people. There are 4 to 5 waiters working at any one time. During weekdays (Sunday to Thursday), there are over 300 customers come to the restaurant and during weekends (Friday and Saturday), over 500 customers at lunch hour 01:00pm-16:30pm. There are several determining factors for a restaurant to be considered a good or a bad one. Taste, cleanliness, the restaurant layout and settings are some of the most important factors. These factors, when managed carefully, will be able to attract plenty of customers [1]. Waiting for service in a restaurant is a part of our daily routine, especially during lunch and dinner time. So, queuing theory is perfectly applicable in restaurant. Researchers have previously used queuing theory to model the restaurant operation [2], reduce cycle time in a busy fast food restaurant [3], as well as to increase throughput and efficiency [4].

\section{Backgrounds}

The queuing theory or waiting line theory was initially proposed by Danish telephone engineer named A. K. Erlang. In the early 1903, he took up the problem on congestion of telephone traffic. The complexity was that during busy periods, telephone operators were unable to handle the calls the moment they were made, resulting in delayed calls. A. K. Erlang directed his first efforts at finding the delay for one operator and later of the results were extended to find the delay for several operators. The field of telephone traffic was further developed by Molins in 1927 and Thornton D-Fry in 1928. It was only after World War II that this early work was extended to other general problems involving queues. His works inspired engineers, mathematicians to deal with queuing problems using probabilistic methods. Queuing theory became a field of applied probability and many of its results have been used in operations research, computer science, telecommunication, traffic engineering and reliability theory. It should be emphasized that is a living branch of science where the experts publish a lot of papers and books.

\section{Basic Characteristics}

The basic characteristics of queuing phenomenon are

$>$ Units arrive at regular or irregular intervals of time, at a given point called the service center. For examples, person arriving a cinema hall, ships arriving a port, patients entering the doctor's chamber and so on. All these units are called the arrivals of customers.

$>$ At a service center there are one or more service channels or service stations. If the service stations are empty, the arriving customers will be served immediately, if not will the arriving customers wait in line until the service is provided. Once the service has been completed the customer leaves the system. 


\section{Characteristics of a Queuing System}

Customer and server are the main elements of queuing model. Customers are normally called units. It may be a person, machine, vehicles and parties etc. Server is the system which performs the services to customers. This may be single or multi-channel. Customers are generated from a source. On arrival at the facility, they can start service immediately or wait in a queue if the facility is busy. When a facility completes a service, it automatically "pulls" a waiting customer, if any, from the queue. If the queue is empty, the facility becomes idle until a new customer arrives.

A queuing model is specified completely by six characteristics:

1. Arrival Fashion: It represents the arriving patterns of customer in the system. Customers don't come at a fixed regular interval of time, their arrivals are random fashion, it tends to be clustered or scattered randomly. In a given time, the number of arrivals is estimated by using a discrete probability distribution (DPD), such as Poisson distribution.

2. Departure (service) Distribution: It represents the patterns in which the number of customer leaves the system. It may also be represented by the service time, which is the time period between successive services. It may be constant or variable but known, or random (variable with only known probability). It is independent of the inter-arrival time. It is described by the exponentially probability distribution.

3. Service Channel: The waiting line system may have multi service channel and single service channel. Arriving customers may form one queue and get serviced, as in a doctor's clinic. The system may have a number of service channels, which may be arranged in parallel or in series or a complex combination of both. A queuing model is called single channel model, when the system has one server only and multichannel model, when the system has a number of parallel channels each with one server.

4. Queue Discipline (service discipline): This represents the order in which customers are selected from a queue, is an important factor in analyzing queuing models. The most common discipline is first come, first serve (FCFS), e.g. railway stations, bank ATM, doctor's clinic etc. Other disciplines are last come, first serve (LCFS) as in big godown and service in random order (SIRO) based on priority.

5. System Capacity: In the system the maximum number of customer can either be finite or infinite. In limited facilities, only a finite number of customers are allowed in the system and new arriving customers are not allowed to join the system unless the number becomes less than the fixed number.

6. Population: The source from which customers are generated may be finite or infinite. A finite source limits the customer's arriving for service.

\section{Kendall's Notation}

In 1953, Kendall proposed a notation for summarizing the characteristics of the queuing situation, which is written as

$$
(a / b / c):(d / e / f)
$$

Where

$a=$ Arrival fashion, $b=$ Service time distribution, $c=$ Number of parallel servers $(1,2,3, \ldots), d=$ Queue discipline, $e=$ Maximum number of units allowed in the system, $f=$ Size of the calling source.

\section{Little's Theorem}

Little's theorem [5] describes the relationship between throughput rate (i.e., arrival and service rate), cycle time and work in process (i.e., number of customers/jobs in the system). This relationship has been shown to be valid for a wide class of queuing models. The theorem states that the expected number of customers $(\mathrm{N})$ for a system in steady state can be determined using the following equation.

$$
L_{s}=\lambda W_{s}
$$

Here, $\lambda$ is the mean arrival rate for customers coming to the system, $W_{s}$ is the expected waiting time in the system. Three fundamental relationships can be derived from Little's theorem [6]:

- $L_{s}$ Increases if $\lambda$ or $W_{s}$ increases.

- $\lambda$ Increases if $L_{s}$ increases or $W_{s}$ decreases.

- $W_{s}$ Increases if $L_{s}$ increases or $\lambda$ decreases. 


\section{Shuruchi Restaurant Model (M/M/1: FCFS/ $\square / \square$ )}

We have obtained one month daily customer's data from this restaurant through interview with the restaurant manager as well as data collections through observations at the restaurant. We have come to know about the capacity of it and the number of waiters and waitress. Based on the above information we have decided that the queuing model that best illustrates the operation of this restaurant is $\mathrm{M} / \mathrm{M} / 1$ : FCFS/ $/ \infty / \infty$.

This means that the arrivals are Poisson distributed and service time is exponentially distributed, restaurant has one server only.

\section{Assumptions:}

$>$ Customers come from an infinite population,

They follow the Poisson distribution,

$>$ Customers behaviour are treated on a service discipline "First Come First Serve" (FCFS) and do not balk or renege,

Dervice time follows the exponential distribution,

$>$ Utilization factor $\rho<1$ that is the average service rate is faster than the average arrival rate.

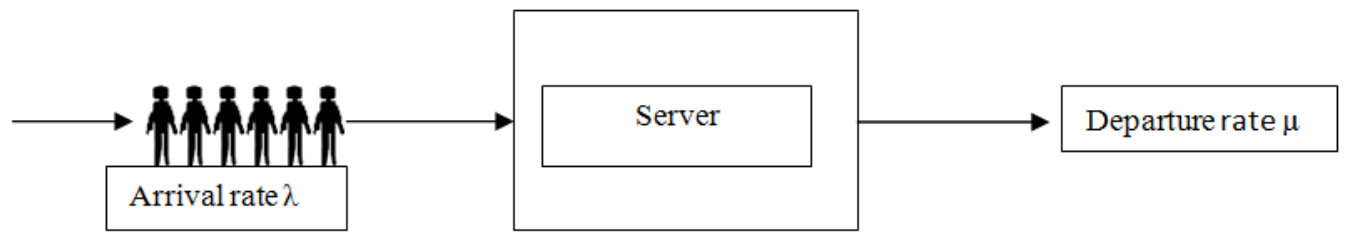

Fig-1: schematic representation of M/M/1 queuing system

For analyzing the Shuruchi restaurant M/M/1 queuing model the following variables will be investigated [6]

○ $\lambda=$ The mean customer's arrival rate.

$\circ \quad \mu=$ The mean service rate.

- $\rho=\frac{\lambda}{\mu} ;$ The utilization factor.

- Probability of zero customers in the restaurant

$p_{0}=1-\rho$

- $\quad p_{n}$ : The probability of having $n$ customers in the restaurant

$p_{n}=p_{0} \rho^{n}=(1-\rho) \rho^{n}$

- $L_{s}$ : Average number of customers in the restaurant

$L_{s}=\frac{\rho}{1-\rho}=\frac{\lambda}{\mu-\lambda}$

- $L_{q}$ :The average number of customers in the queue

$L_{q}=L_{s} \times \rho=\frac{\rho^{2}}{1-\rho}=\frac{\rho \lambda}{\mu-\lambda}$

- $W_{q}$ : The average waiting time in the queue

$W_{q}=\frac{L_{q}}{\lambda}=\frac{\rho}{\mu-\lambda}$

- $W_{s}$ : The average waiting time spent in the restaurant

$$
W_{s}=\frac{L_{s}}{\lambda}=\frac{1}{\mu-\lambda}
$$


VIII. Observation And Discussion

The one month daily customer's data were shared be the restaurant manager, as shown in Table-1

Table-1

\begin{tabular}{|c|l|l|l|l|l|l|l|}
\hline & Sun & Mon & Tue & Wed & Thu & Fri & Sat \\
\hline $1^{\text {st }}$ week & 319 & 309 & 395 & 310 & 375 & 530 & 549 \\
\hline $2^{\text {nd }}$ week & 382 & 335 & 327 & 321 & 331 & 575 & 545 \\
\hline $3^{\text {rd }}$ week & 303 & 349 & 335 & 332 & 323 & 582 & 590 \\
\hline $4^{\text {th }}$ week & 330 & 380 & 349 & 375 & 360 & 603 & 581 \\
\hline
\end{tabular}

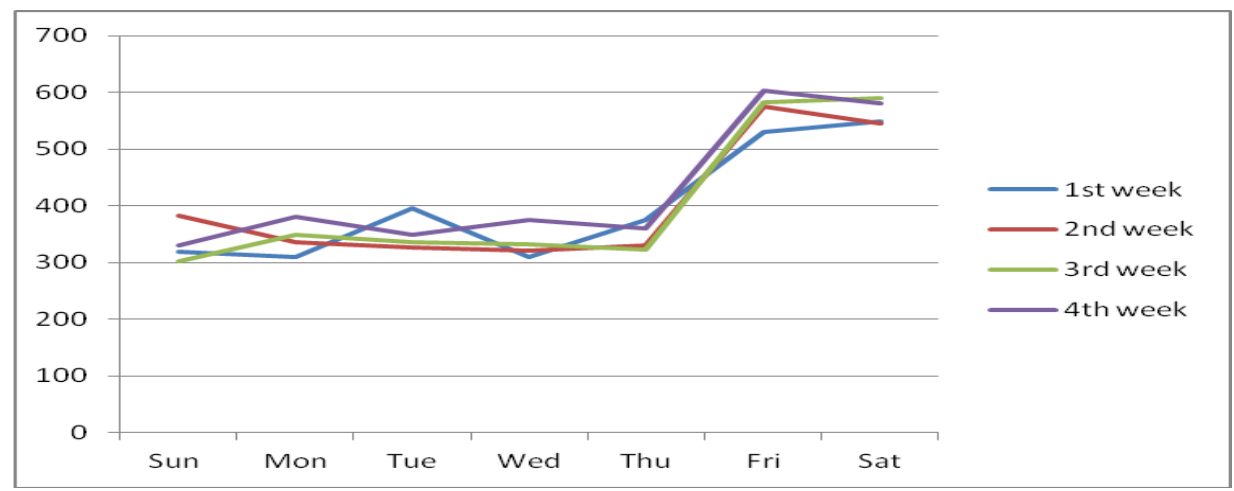

From the above Fig-2, the number of customers on weekend days is greater to the number of customers during weekdays. During lunch time, on weekend, is the busiest period for the restaurant.

\section{Calculation}

There are on average 300 customers come to the restaurant in 3.5 hours during lunch time. So, the arrival rate

$$
\lambda=\frac{300}{210}=1.43 \text { customers per minutes }(\mathrm{cpm}) \text {. }
$$

From the observation and discussion we have found out that on average each customer spends 45 minutes $\left(W_{s}\right)$ in the restaurant, queue length is around 20 customers $\left(L_{q}\right)$, on average and waiting time is around 15 minutes. Now,

$$
W_{q}=\frac{20}{1.43}=13.98 \text { minutes (using "equation 6") }
$$

It is noticed that the actual waiting time does not vary by much compared to the theoretical waiting time.

The average number of customer in the Shuruchi restaurant using Little's theorem (1)

$$
L_{s}=\lambda W_{s}=1.43 \times 45=64.35 \text { customers. }
$$

We can derive the service rate using (4)

$$
\mu=\frac{\lambda\left(1+L_{s}\right)}{L_{s}}=\frac{1.43(1+64.35)}{64.35}=1.45
$$

Hence, $\rho=\frac{\lambda}{\mu}=\frac{1.43}{1.45}=0.98$

With very high utilization rate 0.98 during lunch period, the probability of having zero customer in the system, using (2)

$$
p_{0}=1-\rho=1-0.98=0.02
$$

The queuing theory provides the formula to estimate the probability of having $n$ customers in the restaurant, as follows:

$$
p_{n}=p_{0} \rho^{n}=(1-\rho) \rho^{n}=(1-0.98)(0.98)^{n}=0.02(0.98)^{n}
$$

It is assumed that, impatient customers will start to balk; when they see more than 10 customers are already waiting for getting service in the restaurant. It is also assumed that a patient customer can tolerate maximum 25 customers in the queue. The capacity of the restaurant is 60 customers. Now, we can calculate the probability of having 10 customers in the queue. 
Probability of customers going away $=p$ (more than 10 customers in the queue)

$=p$ (more than 70 customers in the restaurant)

$p_{71-85}=\sum_{n=71}^{85} p_{n}=0.0623=6.23 \%$

\section{Evaluation}

$>$ The utilization factor is directly proportional with the mean number of customers which means that the mean number of customers will increase as the utilization increases.

$>$ In the busy restaurant Shuruchi, the utilization factor is very high 0.98 .

$>$ In case the customers waiting time is lower or in other words we waited for less than 15 minutes the number of customers that are able to be served per minute will increase. When the service rate is higher the utilization will be lower, which makes the probability of the customers going away decreases [1].

\section{Benefits}

Since Shuruchi restaurant is the busiest restaurant, so this research can help this restaurant to ensure their QoS (Quality of Service) by forecasting, if there are many customers in the queue. It may become a model to analyze the current situation and to improve the system as restaurant can now, estimate how many customers will wait in the queue, by anticipating the huge number of customers coming to the restaurant with a view to getting services. The formulas provide mechanism to model the restaurant queue that is simpler than the creation of simulation model [7]

\section{Conclusion}

This research paper has discussed the application of single channel M/M/1 queuing model in Shuruchi restaurant. Applying this model we have obtained that, the customer's arrival rate is $1.43 \mathrm{cpm}$ and the service rate is $1.45 \mathrm{cpm}$. If there are 10 customers in the queue waiting for service, the probability of buffer flow is $6.23 \%$. The probability of buffer overflow is the probability that customers will run away, because may be they are impatient to wait in the queue [1]. We expect our research can help doing the betterment of the restaurant.

As our future works, we will develop a simulation model for the restaurant. By developing a simulation model we will be able to confirm the results of the analytical model that we develop in this paper. In addition, a simulation model allows us to add more complexity so that the model can mirror the actual operation of the restaurant more closely [8]

[1] Mathias Dharmawirya, Erwin Adi, "Case Study for Restaurant Queuing Model", 2011 International Conference on Management and Aritificial Intelligence, IPEDR vol.6, Indonesia.

[2] D.M. Brann and B.C. Kulick, "Simulation of restaurant operations using the Restaurant Modeling Studio," Proceedings of the 2002 Winter Simulation Conference, IEEE Press, Dec. 2002, pp. 1448-1453.

[3] S. A. Curin, J. S. Vosko, E. W. Chan and O. Tsimhoni," Reducing Service Time at a Busy Fast Food Restaurant on Campus," Proceedings of the 2005 Winter Simulation Conference, IEEE Press, Dec. 2005.

[4] A. K. Kharwat," Computer Simulation: an Important Tool in the Fast Food Industry," Proceedings of the 1996 Winter Simulation Confernce, IEEE Press, Dec. 1996, pp.1264-1271.

[5] J. D.C. Little, “A Proof for the Queuing Formula: $L=\lambda W$,” Operations Research, vol. 9(3), 1961, pp. 383-387.

[6] M. Laguna and J. Marklund, Business Process Modeling, Simulation and Design. ISBN 0-13-091519-X. Pearson Prenice Hall, 2005.

[7] T. C. Whyte and D. W. Starks, "ACE: A Decision Tool Restaurant Managers," Proceedings of the 1996 Winter Simulation Conference, IEEE Press, Dec. 1996, pp. 1257-1263.

[8] T. Altiok and B. Melamed, Simulation Modeling and Analysis with ARENA. ISBN 0-12-370523. Academic Press, 2007.

[9] V. K.. Kapoor (July, 1999), Operations Research (4 $4^{\text {th }}$ Edition).

[10] Cooper R. B. (1980), Introduction to queuing theory (2 $1^{\text {th }}$ Edition). North Holland.

[11] H. A. Taha (2007), Operations Research ( $8^{\text {th }}$ Edition).

[12] Frederick S. Hillier \& Mark S. Hiller (2005), Introduction to Management Science. A Modeling and Case Studies Approach with Spreadsheets.

[13] Prem Kumar Gupta \& D. S. Hira (2003), Operations Research.

[14] Chase R. ET Aquilano N. (1973), Production and Operations Management. Irawn.

[15] Lee A. M. (1971), Applied Queuing Theory. New York.

[16] Morse Phillip. (1971), Methods of Operations Research. London.

[17] Wagner H. M. (1975), Principles of Operations Research. International Edition.

[18] Winston Wayane L. (1991), Operations Research, Applications and Algorithms ( $2^{\text {th }}$ Edition).

[19] Huliand Tao Yang, (2000), "Theory and methodology queues with a variable number of servers," European Journal of Operations Research, vol. 124, pp. 615-628.

[20] N. K. Tiwari \& Shishir K. Shandilya. (2009), Operations Research ( $3^{\text {rd }}$ Edition).

[21] Nita H. Shaha, Ravi M. Gor, Hardik Soni. (2010), Operations Research ( $3^{\text {rd }}$ Edition).

[22] S. K. Dhar, Tanzina Rahman, (2013), "Case Study of Bank ATM Queuing Model," IOSR Journal of Mathematics, IOSR-JM, 7(1), 01-05. 
[23] V. S. Selvi and M. Nishanthi,"Mathematical Appliations of Queuing Theory in Call Centers,"International Journal of Scientific \& Engineering Research, IJSER, vol. 3, ISSUE 11, November-2012.

[24] Bhavin Patel Parvin Bhathawala, (2012),"Case Study for Bank ATM Queuing Model, International Journal of Engineering Research and Applications," IJERA, 2(5), 1278-1284, ISSN: 2248-9622.

[25] M. S. R. Murthy, M. Pushpa Latha \& K.G. R. Deepthi (January-14),"Minimizing The Waiting Time at Bank ATM for service with Queuing Model,"International Journal of Scientific Research, IJSR, and vol. 3, ISSN: 22277-8179.

[26] Mohammad Shyfur Rahman Chowdhury,"Queuing Theory Model Used to Solve The Waiting Line of a Bank- A Study on Islami Bank Bangladesh Limited, Chawkbazar Branch, Chittagong,"Asian Journal of Social Sciences \& Humanities, vol. 2 No. 3 August 2013. 